Ann. Biol. anim. Bioch. Biophys., I972, 12 (3), 43I-440.

\title{
ÉVOLUTION DES TAUX PLASMATIQUES D'ACIDES GRAS LIBRES \\ ET DES SUCRES RÉDUCTEURS \\ LORS D'UN TRAITEMENT \\ AUX HORMONES SOMATOTROPES PORCINE ET BOVINE CHEZ LE LAPIN HYPOPHYSECTOMISÉ
}

\author{
A. VÉZINHET, J. CHARRIER et L. DAUZIER \\ Station de Physiologie animale, \\ École nationale supérieure agronomique \\ Centre de Recherches, I. N. R. A., \\ 34000 Montpellier
}

avec la collaboration technique de E. Bouthier, Y. Carles et Thérèse Chery

\section{RÉSUMÉ}

L'influence d'injections répétées de BGH et PGH sur l'évolution des taux plasmatiques d'acides gras libres et des glucides a été étudiée sur des lapines hypophysectomisées. Les dosages des métabolites étaient pratiqués aux temps o heure avant le traitement puis $1,2,5$ et 9 heures, après l'injection de l'hormone considérée, sur des échantillons de sang veineux. Les résultats suivants ont été observés.

- Glycémie : l'administration de PGH entraîne chez le Lapin hypophysectomisé nourri ad libitum un effet hypoglycémiant qui se manifeste lors des première à deuxième heures après l'injection. Cet effet n'est pas suivi d'une hyperglycémie. Avec la BGH les variations observées vont dans le même sens, mais avec une intensité moindre.

- Taux d'acides gras libres : avec la PGH une élévation importante est observée aux temps $I$ et 2 heures après l'injection, et le taux redevient progressivement normal au bout de 9 heures. La BGH n'a pas manifesté d'effet particulier. Lors du sacrifice de certains animaux les dépôts adipeux de réserve, évalués à partir du gras périrénal, sont apparus nettement réduits chez les lapines hypophysectomisées traitées à la $\mathrm{PGH}$ par rapport aux témoins hypophysectomisées traitées au ClNa 9 p. I ooo.

Aucune variation sur la croissance pondérale globale n'a été enregistrée.

I.e taux plasmatique des acides gras libres est influencé par un grand nombre de facteurs tels que l'état nutritionnel, le niveau de glycémie et divers mécanismes neuro-humoraux parmi lesquels 1'hormone somatotrope joue un rôle particulièrement 
important. RABEN et HOLLENBERG (I958) ont été les premiers à mettre en évidence chez le Chien l'effet lipolytique de GH de différentes espèces. ENGEL et al. (I958) ont signalé une élévation importante de la concentration plasmatique en acides gras libres après une injection intrapéritonéale de $\mathrm{GH}$ à des rats hypophysectomisés nourris ad libitum. KNOBIL et GREEP (I959) travaillant chez le Singe Rhésus ont montré qu'une simple injection de $\mathrm{GH}$ de singe faisait accroître le taux plasmatique des acides gras libres chez l'animal hypophysectomisé ou intact. Cependant, l'effet était plus net lorsque les animaux étaient à jeun. ENGEI, et al. (I959) ont observé chez 1'Homme que l'élévation des acides gras libres plasmatiques survenait 6 heures après une injection de $\mathrm{GH}$ humaine ce qui était en accord avec les observations faites par GREENBAUM et GLASCOCK (I957) sur le Rat. RABEN (I959) quant à lui, signalait que les teneurs en acides gras libres observées après 24 heures de jeûne étaient doublées ou triplées 4 heures après un traitement à l'hormone somatotrope humaine, cet effet de la GH pouvant être supprimé par un apport en sucre ou en nourriture. WINKLER et al. (I962) ont observé une élévation du taux plasmatique des acides gras libres chez le Chien 3 heures après injection de GH bovine, tandis que AltSZULLER et al. (I968), toujours chez le Chien intact, ont montré que cette élévation survenait dans les 3 à 9 heures après l'administration de $\mathrm{GH}$ bovine. Dans le même temps, apparaissaient un " turn over " et une oxydation accrus des acides gras libres. Le déclin de l'effet de la GH sur le "turn over " des acides gras libres dès les $I 7^{\mathrm{e}}$ à $20^{\circ}$ heures après l'injection est accompagné d'une diminution semblable des effets aigus de l'hormone somatotrope sur le métabolisme des acides gras libres. Si le traitement est maintenu à raison de I mg de GH par $\mathrm{kg}$ et par jour, le taux des acides gras libres revient à un niveau normal dans un laps de temps de l'ordre de 7 jours, l'apport de nourriture aux animaux n'ayant pas d'incidence sur le déroulement du phénomène de mobilisation des lipides, mais seulement sur 1'amplitude de la réponse. Cette diminution de l'effet de la GH lors d'un traitement chronique pourrait être liée à la très forte spécificité de 1'hormone somatotrope due à son haut pouvoir antigénique signalé par de nombreux auteurs, notamment par Goodman et KnobIL, (I959) lors d'une étude de l'action de différentes GH sur la mobilisation des acides gras libres chez le Singe. RABEN (I959), travaillant chez 1'Homme, a également montré que les GH de Singe et d'Homme sont efficaces sur la mobilisation des acides gras libres, tandis que les $\mathrm{GH}$ porcine $(\mathrm{PGH})$ et bovine $(\mathrm{BGH})$ sont inactives. Ces différences d'activité biologique pourraient être liées à des particularités d'ordre physico-chimique comme cela a été signalé par WILHELMI (I955).

D'autre part, il semble que l'on puisse établir des parentés de type immunochimique entre GH d'espèces différentes : dans un système d'immunodiffusion sur gel d'agar on a noté l'existence de réactions croisées, à différents degrés, entre les GH de bovins, ovins et porcins et un sérum anti GH Iapin. En particulier, la réaction croisée était beaucoup plus intense dans le système PGH-sérum anti GH Lapin que dans le système BGH-sérum anti GH Lapin (ELLIs et al., I968), mais aucune ligne de précipitation n'est apparue dans le système GH primate-sérum anti GH Lapin. Cette parenté immunologique plus ou moins étroite nous a conduit à entreprendre l'étude comparative d'un traitement avec la PGH et la BGH sur la mobilisation des lipides et l'évolution de la glycémie du lapin hypophysectomisé. 


\title{
MATÉRIEL ET MÉTHODES
}

\begin{abstract}
Ce travail a porté sur I6 lapines hypophysectomisées à l'âge de Ioo jours dont les poids étaient compris entre 2500 et $3500 \mathrm{~g}$ au moment de l'intervention. L'hypophysectomie a été réalisée par voie parapharyngienne selon la technique de JacoBsohn et Westman. Les animaux opérés étaient maintenus sans traitement jusqu'à un âge de 125 jours dans des conditions précédemment décrites (VÉzINHET, r968). 5 lapines ont été ensuite traitées avec une hormone somatotrope porcine, dont deux avec une hormone PGH-NIH (lot NIH-P-5or B activité I UI par mg), et 3 avec une PGH commerciale (N. B. C., 7433, 5o UI). 5 autres lapines ont subi un traitement avec une hormone bovine (BGH Byla, lot oI-69, activité $\mathrm{r}$, I UI par $\mathrm{mg}$ ), les 6 lapines restantes servaient de témoins.

Les hormones somatotropes bovine et porcine étaient injectées par voie intraveineuse à la dose de I UI par $\mathrm{kg}$ de poids vif. Le traitement hormonal commencé le $125^{\mathrm{e}}$ jour de la vie postnatale était poursuivi jusqu'au $I 37^{e}$ jour au rythme d'une injection tous les deux jours ; les animaux étaient nourris ad libitum pendant toute la durée de l'expérience. Les échantillons de sang étaient prélevés à la veine marginale de l'oreille, au temps o précédant le traitement, puis à I, 2, 5 et 9 heures après l'administration de l'hormone. Pour les témoins hypophysectomisés qui recevaient une injection de sérum physiologique, seuls les prélèvements aux temps o, 5 et 9 heures ont été effectués, après avoir vérifié lors de dosages préliminaires que les temps $I$ et 2 heures n'apportaient aucune information particulière. Les solutions d'hormones somatotropes utilisées étaient préparées chaque fois par dilution dans l'eau distillée à raison de I UI par ml. L'absence de reliquats hypophysaires chez les animaux ayant subi l'ablation de la glande pituitaire a été contrôlée histologiquement par l'examen en coupes sériées de pièces anatomiques comprenant l'ensemble hypothalamus-selle turcique.

Les mesures des concentrations plasmatiques en glucides réducteurs ont été réalisées par la technique de NeLson (I944) et celles des acides gras libres par la méthode de DoLE et MEINERTZ (I960).

L'analyse des résultats a consisté en une simple comparaison entre valeurs absolues obtenues pour les animaux traités (BGH ou PGH) et leurs témoins hypophysectomisés. En raison des corrélations qui existent entre les valeurs obtenues à des temps successifs, aucune analyse statistique rigoureuse n'a pu être mise en place dans le cadre de cette étude.
\end{abstract}

\section{RÉSULTATS}

\section{Infuence des hormones somatotropes porcine ( $\mathrm{PGH}$ ) et bovine (BGH)}

\section{Sur le taux plasmatique en glucides.}

Du tableau I il ressort que une ou deux heures après l'administration de PGH on note une chute du taux plasmatique en glucides réducteurs. Celle-ci est particulièrement nette lors de la première injection (fig. I) pour laquelle on enregistre au temps 2 heures une diminution de $3^{8} \mathrm{p}$. Ioo par rapport au temps o. A la $2^{\mathrm{e}}$ injection le pourcentage maximum de chute de la glycémie, observé cette fois au temps I heure, n'est plus que de $3 \mathrm{I}$ p. IOO par rapport à la valeur initiale avant traitement. Pour les injections suivantes les maxima de chute de la teneur en glucides du plasma après administration de $\mathrm{PGH}$ restent de l'ordre de 20 à $25 \mathrm{p}$. Ioo.

La glycémie des animaux traités à la $\mathrm{BGH}$ accuse également une diminution après l'administration de l'hormone, mais contrairement à celle des animaux traités $\mathrm{PGH}$, cette diminution est toujours comprise entre ro et $16 \mathrm{p}$. Ioo. Au temps 9 heures après l'injection, on enregistre d'une manière générale des glycémies du même ordre 


\section{TABLEAU I}

Comparaison des taux plasmatiques moyens en glucides

(mg/roo ml de plasma) 士 l'écart-type, obtenus pour des lapines hypophysectomisées traitées à l'hormone somatotrope porcine (PGH) ou bovine (BGH)

et pour des témoins hypophysectomisées recevant ClNa 9 p. 1000

\begin{tabular}{|c|c|c|c|c|c|c|c|}
\hline $\begin{array}{c}\text { Jours } \\
\text { d'injection }\end{array}$ & $\begin{array}{c}\text { Nature } \\
\text { du } \\
\text { traitement }\end{array}$ & $0 \mathrm{~h}$ & $1 \mathrm{~h}$ & $2 \mathrm{~h}$ & $5 \mathrm{~h}$ & $9 \mathrm{~h}$ & $\frac{\mathrm{H}_{0}-\mathrm{H}_{1}{ }^{*}}{\mathrm{H}^{0}} \times 100$ \\
\hline $\begin{array}{c}1 \text { re } \\
\text { injection }\end{array}$ & $\begin{array}{c}\text { PGB } \\
\text { BGH } \\
\text { T }\end{array}$ & $\begin{array}{r}99 \pm 13 \\
128 \pm 18 \\
137 \pm 13\end{array}$ & $\begin{array}{r}67 \pm 16 \\
113 \pm 27\end{array}$ & $\begin{array}{r}61 \pm 9 \\
123 \pm 27\end{array}$ & $\begin{array}{r}65 \pm 21 \\
123 \pm 25 \\
136 \pm 12\end{array}$ & $\begin{array}{r}78 \pm 13 \\
137 \pm 22 \\
136 \pm 9\end{array}$ & $\begin{array}{l}38 \\
12\end{array}$ \\
\hline $2^{\mathrm{e}}$ & $\begin{array}{c}\text { PGH } \\
\text { BGH } \\
\quad T\end{array}$ & $\begin{array}{r}93 \pm 33 \\
101 \pm 31 \\
114 \pm 34\end{array}$ & $\begin{array}{l}64 \pm 30 \\
91 \pm 29\end{array}$ & $\begin{array}{r}69 \pm 41 \\
106 \pm 35\end{array}$ & $\begin{array}{r}85 \pm 44 \\
120 \pm 8 \\
124 \pm 35\end{array}$ & $\begin{array}{r}91 \pm 30 \\
108 \pm 24 \\
106 \pm 37\end{array}$ & $\begin{array}{l}31 \\
10\end{array}$ \\
\hline $\begin{array}{c}3^{e} \\
\text { injection }\end{array}$ & $\begin{array}{c}\text { PGH } \\
\text { BGH } \\
\quad T\end{array}$ & $\begin{array}{l}120 \pm 18 \\
121 \pm 18 \\
130 \pm 16\end{array}$ & $\begin{array}{r}93 \pm 36 \\
102 \pm 26\end{array}$ & $\begin{array}{r}93 \pm 42 \\
111 \pm 10\end{array}$ & $\begin{array}{l}114 \pm 16 \\
130 \pm 14 \\
131 \pm 12\end{array}$ & $\begin{array}{l}120 \pm 13 \\
139 \pm 15 \\
133 \pm 21\end{array}$ & $\begin{array}{l}22 \\
16\end{array}$ \\
\hline$\stackrel{4^{\mathrm{e}}}{\text { injection }}$ & $\begin{array}{c}\text { PGH } \\
\text { BGH } \\
\text { T }\end{array}$ & $\begin{array}{l}131 \pm 15 \\
130 \pm 6 \\
135 \pm 17\end{array}$ & $\begin{array}{l}102 \pm 27 \\
110 \pm 14\end{array}$ & $\begin{array}{r}95 \pm 27 \\
113 \pm 13\end{array}$ & $\begin{array}{l}137 \pm 25 \\
106 \pm 21 \\
140 \pm 19\end{array}$ & $\begin{array}{l}144 \pm 41 \\
129 \pm 19 \\
142 \pm 25\end{array}$ & $\begin{array}{l}27 \\
15\end{array}$ \\
\hline $\begin{array}{c}5^{\mathrm{e}} \\
\text { injection }\end{array}$ & $\begin{array}{c}\text { PGH } \\
\text { BGH } \\
\text { T }\end{array}$ & $\begin{array}{l}103 \pm 33 \\
112 \pm 9 \\
125 \pm 29\end{array}$ & $\begin{array}{r}89 \pm 36 \\
101 \pm 26\end{array}$ & $\begin{array}{r}80 \pm 34 \\
108 \pm 15\end{array}$ & $\begin{array}{l}100 \pm 39 \\
115 \pm 28 \\
123 \pm 21\end{array}$ & $\begin{array}{l}101 \pm 41 \\
142 \pm 5 \\
115 \pm 19\end{array}$ & $\begin{array}{l}22 \\
10\end{array}$ \\
\hline $\begin{array}{c}6^{\mathrm{e}} \\
\text { injection }\end{array}$ & $\begin{array}{c}\text { PGH } \\
\text { BGH } \\
\text { T }\end{array}$ & $\begin{array}{l}113 \pm 18 \\
116 \pm 21 \\
117 \pm 32\end{array}$ & $\begin{array}{r}91 \pm 33 \\
100 \pm 31\end{array}$ & $\begin{array}{r}90 \pm 33 \\
119 \pm 34\end{array}$ & $\begin{array}{l}112 \pm 27 \\
126 \pm 25 \\
123 \pm 26\end{array}$ & $\begin{array}{l}118 \pm 22 \\
128 \pm 21 \\
123 \pm 15\end{array}$ & $\begin{array}{l}20 \\
14\end{array}$ \\
\hline $\begin{array}{c}7^{\mathrm{e}} \\
\text { injection }\end{array}$ & $\begin{array}{c}\text { PGH } \\
\text { BGH } \\
\text { T }\end{array}$ & $\begin{array}{l}110 \pm 24 \\
107 \pm 27 \\
115 \pm 20\end{array}$ & $\begin{array}{l}82 \pm 28 \\
94 \pm 35\end{array}$ & $\begin{array}{l}91 \pm 25 \\
93 \pm 39\end{array}$ & $\begin{array}{l}101 \pm 13 \\
108 \pm 34 \\
111 \pm 32\end{array}$ & $\begin{array}{l}122 \pm 7 \\
119 \pm 11 \\
129 \pm 17\end{array}$ & $\begin{array}{l}25 \\
13\end{array}$ \\
\hline $\begin{array}{r}* \mathrm{H}_{0}= \\
\mathrm{H}_{1}= \\
\frac{\mathrm{H}_{0}-}{\mathrm{H}_{0}}\end{array}$ & $\begin{array}{l}=\text { Glycémie } \\
=\text { Glycémie } 1 \\
\underline{\mathrm{H}_{\mathrm{I}}} \times 100=\end{array}$ & temps 0 . & theng & l'adminis & ion de $G$. & tion. & \\
\hline
\end{tabular}

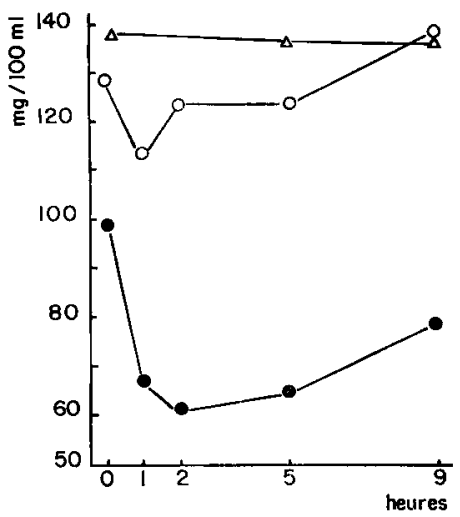

FIG. I. - Évolution de la glycémie (mg/roo ml de plasma) obtemue chez des lapines hypophysectomisées au 1 er jour d'un traitement chronique à la $P G H(\bullet-\bullet)$ et à la $B G H(\circ-\infty)$

Lapines témoins hypophysectomisées recevant $\mathrm{ClNa}(9 \mathrm{p}$. I ooo) $(\Delta-\Delta)$ 
que celles du temps o. Même quand les valeurs du temps 9 heures sont supérieures à celles observées avant le traitement hormonal, elles le sont faiblement et en aucun cas nous n'avons pu mettre en évidence une hyperglycémie consécutive à l'hypoglycémie. On ne peut également parler ici d'effet diabétogène de 1'hormone de croissance car les valeurs des glycémies relevées au temps o du premier au $7^{\mathrm{e}}$ jour de traitement sont toujours sensiblement comparables; même dans le cas du lot des animaux traités à la $\mathrm{PGH}$ qui passe d'une valeur moyenne initiale en glycémie de 99 le premier jour d'injection à une valeur de I2O, puis I $3 \mathrm{I}$, les $3^{\mathrm{e}}$ et $4^{\mathrm{e}}$ jours, on ne peut conclure à un effet diabétogène en raison de l'importante variabilité des résultats dont témoignent les écarts-types élevés. Enfin, pour les animaux témoins on n'observe jamais de variation importante de la glycémie entre les temps o, 5 et 9 heures.

\section{Sur la mobilisation des lipides de réserve.}

Les lapines hypophysectomisées traitées à la PGH montrent une très forte élévation de leurs taux plasmatiques en acides gras libres. Les valeurs maximales moyennes sont toujours observées au temps I heure après le traitement hormonal (tabl. 2) (fig. 2). Les pourcentages d'augmentation par rapport au temps o sont compris entre $47^{2} \mathrm{p}$. Ioo lors de la $2^{\mathrm{e}}$ injection et $787 \mathrm{p}$. Ioo lors de la $6 \mathrm{e}$ injection. Au temps 2 heures après l'administration de $\mathrm{PGH}$ les valeurs en acides gras libres du plasma sont encore très fortes et voisines de celles du temps I heure. Il faut attendre le temps 5 heures pour observer une nette diminution de ces valeurs. Enfin au temps 9 heures on a dans la plupart des cas, retrouvé un niveau moyen en acides gras libres voisin de celui du temps o.

En ce qui concerne les animaux hypophysectomisés traités à la BGH, le taux moyen en acides gras libres du plasma ne varie guère au cours des 5 prélèvements effectués pour chaque jour d'injection. On relève néanmoins des variations individuelles importantes notamment lors des temps I, 2 et 5 heures pour lesquels on note des écart-types très élevés.

Les lapines témoins hypophysectomisées n'ont jamais subi de variations notables de leurs taux en acides gras libres plasmatiques.

Afin d'étudier l'influence du traitement à l'hormone somatotrope sur les réserves adipeuses, nous avons procédé au sacrifice de certains animaux après la $7^{\mathrm{e}}$ injection, et pesé leur tissu adipeux périrénal. Pour 3 lapines traitées $\mathrm{PGH}$ on observe des valeurs de I4,6 g, $24 \mathrm{~g}$ et I5,8 g, soit une moyenne de $\mathrm{I} 8 \mathrm{~g}$.

Pour 4 lapines témoins hypophysectomisées on relève des valeurs de $65.5 \mathrm{~g}$, $82 \mathrm{~g}, 7^{2} \mathrm{~g}$, et $72,5 \mathrm{~g}$, soit une moyenne de $73 \mathrm{~g}$. Cette donnée confirme clairement les effets de l'hormone somatotrope sur la mobilisation des lipides de réserve.

Enfin, les contrôles histologiques effectués sur des pièces anatomiques comprenant l'ensemble hypothalamus-selle turcique, révèlent une absence totale de reliquats hypophysaires pour les lapines traitées, soit avec la PGH, soit avec la BGH. Il en est de même pour 4 des lapines témoins, chez les deux autres nous avons trouvé au contact de l'hypothalamus quelques amas de cellules semblant appartenir à la pars tuberalis. 


\section{TABLEAU 2}

Comparaison des taux plasmatiques moyens en acides gras libres $(\mu \mathrm{Eq} / 1$ de plasma) \pm l'écart-type, obtenus pour des lapines hypophysectomisées traitées à l'hormone somatotrope porcine (PGH) ou bovine (BGH) et pour des témoins hypophysectomisées recevant ClNa 9 p. 1000

\begin{tabular}{|c|c|c|c|c|c|c|c|}
\hline $\begin{array}{c}\text { Jours } \\
\text { d'injection }\end{array}$ & $\begin{array}{c}\text { Nature } \\
\text { du } \\
\text { traitement }\end{array}$ & $0 \mathrm{~h}$ & $1 \mathrm{~h}$ & $2 \mathrm{~h}$ & $5 \mathrm{~h}$ & $9 \mathrm{~h}$ & $\frac{\mathrm{H}_{1}-\mathrm{H}_{0}{ }^{*}}{\mathrm{H}_{0}} \times 100$ \\
\hline $\begin{array}{c}1 \text { re } \\
\text { injection }\end{array}$ & $\begin{array}{c}\text { PGH } \\
\text { BGH } \\
\text { T }\end{array}$ & $\begin{array}{l}392 \pm 90 \\
333 \pm 137 \\
937 \pm 54\end{array}$ & $\begin{array}{c}2794 \pm 675 \\
327 \pm 80\end{array}$ & $\begin{array}{c}2469 \pm 248 \\
302 \pm 54\end{array}$ & $\begin{array}{c}1468 \pm 308 \\
222 \pm 63 \\
278 \pm 162\end{array}$ & $\begin{array}{l}395 \pm 114 \\
196 \pm 118 \\
148 \pm 58\end{array}$ & 613 \\
\hline $\begin{array}{c}2^{\mathbf{e}} \\
\text { injection }\end{array}$ & $\begin{array}{c}\text { PGH } \\
\text { BGH } \\
\quad T\end{array}$ & $\begin{array}{l}379 \pm 96 \\
330 \pm 7 \\
287 \pm 185\end{array}$ & $\mid \begin{array}{c}2169 \pm 190 \\
278 \pm 77\end{array}$ & $\begin{array}{r}2195 \pm 506 \\
331 \pm 189\end{array}$ & $\mid \begin{array}{l}1158 \pm 276 \\
240 \pm 76 \\
286 \pm 167\end{array}$ & $\left|\begin{array}{l}581 \pm 278 \\
307 \\
277 \\
243\end{array}\right|$ & 472 \\
\hline injection & $\begin{array}{c}\text { PGH } \\
\text { BGH } \\
\quad \mathrm{T}\end{array}$ & $\left|\begin{array}{l}435 \pm 170 \\
264 \pm 45 \\
257 \pm 86\end{array}\right|$ & $\begin{array}{r}2492 \pm 475 \\
451 \pm 191\end{array}$ & $\begin{array}{r}2336 \pm 494 \\
458 \pm 187\end{array}$ & $\left|\begin{array}{c}1433 \pm 871 \\
349 \pm 163 \\
234 \pm 88\end{array}\right|$ & $\begin{array}{l}847 \pm 663 \\
312 \pm 124 \\
179 \pm 80\end{array}$ & 473 \\
\hline $\begin{array}{c}4^{e} \\
\text { injection }\end{array}$ & $\begin{array}{c}\mathrm{PGH} \\
\mathrm{BGH} \\
\quad \mathrm{T}\end{array}$ & $\begin{array}{l}314 \pm 62 \\
234 \pm 59 \\
266 \pm 83\end{array}$ & $\begin{array}{r}2356 \pm 217 \\
357 \pm 208\end{array}$ & $\left\{\begin{array}{r}1922 \pm 762 \\
451 \pm 256\end{array}\right.$ & $\mid \begin{array}{c}1077 \pm 47 \\
318 \pm 184 \\
243 \pm 50\end{array}$ & $\begin{array}{l}594 \pm 569 \\
194 \pm 36 \\
249 \pm 68\end{array}$ & 650 \\
\hline $\begin{array}{c}5^{\mathbf{e}} \\
\text { injection }\end{array}$ & $\begin{array}{c}\text { PGH } \\
\text { BGH } \\
\text { T }\end{array}$ & $\begin{array}{l}223 \pm 47 \\
213 \pm 34 \\
325 \pm 175\end{array}$ & $\begin{array}{r}1937 \pm 521 \\
351 \pm 159\end{array}$ & $\left|\begin{array}{c}1707 \pm 478 \\
202 \pm 54\end{array}\right|$ & $\begin{array}{l}570 \pm 375 \\
240 \pm 262 \\
215 \pm 131\end{array}$ & $\begin{array}{l}347 \pm 168 \\
118 \pm 30 \\
298 \pm 184\end{array}$ & 769 \\
\hline $\begin{array}{c}6^{\mathrm{e}} \\
\text { injection }\end{array}$ & $\begin{array}{c}\text { PGH } \\
\text { BGH } \\
\mathrm{T}\end{array}$ & $\begin{array}{l}236 \pm 42 \\
219 \pm 75 \\
249 \pm 50\end{array}$ & $\begin{array}{r}2093 \pm 502 \\
331 \pm 248\end{array}$ & $\begin{array}{r}1674 \pm 696 \\
300 \pm 209\end{array}$ & $\begin{array}{c}1249 \pm 966 \\
361 \pm 204 \\
241 \pm 36\end{array}$ & $\begin{array}{l}316 \pm 109 \\
228 \pm 60 \\
256 \pm 113\end{array}$ & 787 \\
\hline $\begin{array}{c}7^{\mathrm{e}} \\
\text { injection }\end{array}$ & $\begin{array}{c}\text { PGH } \\
\text { BGH } \\
\text { T }\end{array}$ & $\begin{array}{l}250 \pm 46 \\
239 \pm 65 \\
320 \pm 163\end{array}$ & $\begin{array}{r}2139 \pm 132 \\
314 \pm 152\end{array}$ & $\begin{array}{r}1788 \pm 236 \\
365 \pm 106\end{array}$ & $\begin{array}{r}1213 \pm 387 \\
431 \pm 298 \\
355 \pm 157\end{array}$ & $\begin{array}{l}287 \pm 74 \\
236 \pm 29 \\
359 \pm 163\end{array}$ & 756 \\
\hline
\end{tabular}

* $\mathrm{H}_{0}=$ Acides gras libres plasmatiques à $0 \mathrm{~h}$. $\mathrm{H}_{1}=$ Acides gras libres plasmatiques à $1 \mathrm{~h}$.

$\frac{\mathrm{H}_{\mathbf{1}}-\mathrm{H}_{\mathbf{0}}}{\mathrm{H}_{0}} \times 100=$ p. 100 d'augmentation en acides gras libres au temps $1 \mathrm{~h}$ pour les animaux traités PGH.

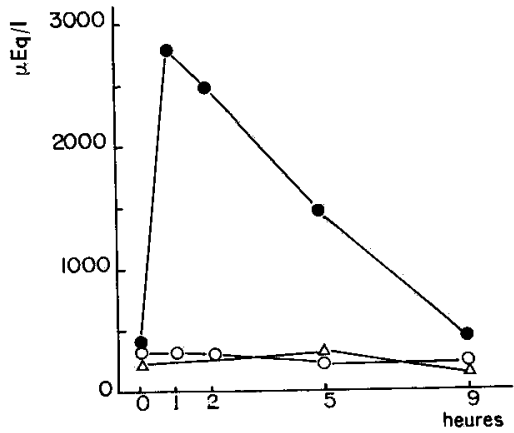

FIG. 2. - Evolution du taux plasmatique moyen en acides gras libres $(\mu \mathrm{Ep} / \mathrm{l}$ de plasma) obtenue chez des lapines hypophysectomisées

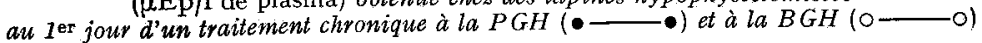

Lapines hypophysectomisées recevant CINa 9 p. I ooo $(\Delta-\Delta)$ 


\section{DISCUSSION}

Ces résultats soulignent le rôle que joue 1'hormone de croissance dans la régulation du métabolisme des substances non azotées chez le Lapin.

Pour les lipides ils confirment l'effet lipolytique de 1'hormone somatotrope, ceci uniquement dans le cas de 1'hormone d'origine porcine.

Le fait que la $\mathrm{BGH}$ se soit révélée inefficace pourrait être expliqué sur la base des observations faites par EisLIs et al. (Ig68) qui avaient signalé une parenté immunologique plus étroite entre les GH de lapin et de porc qu'entre celles de lapin et de bovin. Dans le cas des animaux traités PGH on note que l'effet lipolytique est durable et se manifeste jusqu'à 5 heures après l'administration de 1'hormone. L/e déclin des effets de la $\mathrm{PGH}$ apparait nettement à la $9^{\mathrm{e}}$ heure donc beaucoup plus précocement que ne le signalaient AlTszULLER et al. (I968) chez le Chien pour lequel il fallait attendre les $I 7^{\mathrm{e}}$ à $20^{\mathrm{e}}$ heures pour observer ce phénomène. De plus ces mêmes auteurs notaient une diminution de l'effet de la GH lors d'un traitement chronique. Nous n'avons pour notre part rien observé de semblable, puisque le pourcentage d'augmentation du taux plasmatique moyen après traitement reste du même ordre de la première à $1 \mathrm{a} 7^{\mathbf{e}}$ injection.

Pour les animaux traités BGH le seul fait qui mérite d'être commenté concerne l'augmentation des écart-types des moyennes observées pour les différents temps de prélèvement. Nous avons déjà signalé qu'ils sont d'une manière générale beaucoup plus élevés pour les temps $\mathrm{r}, 2$ et 5 heures par rapport aux temps o et 9 heures. Cette observation qui se vérifie toujours pour les $3^{\mathrm{e}}$ à $7^{\mathrm{e}}$ injections pourrait indiquer que la manipulation des animaux dans des intervalles de temps trop rapprochés conduit à un effet " stress " qui se manifeste par une augmentation des acides gras libres résultant de la mobilisation des graisses. De nombreux auteurs ont déjà signalé un tel effet.

L'évolution des glucides réducteurs du plasma après administration de $\mathrm{GH}$ nous apporte un certain nombre d'enseignements. On a pu notamment observer un effet hypoglycémiant qui se manifeste très nettement lors des première et deuxième injections pour les animaux traités à la PGH. Cet effet persiste de la $3^{\mathrm{e}}$ à la $7^{\mathrm{e}}$ injection avec une intensité légèrement réduite. Pour les animaux traités à la $\mathrm{BGH}$ on observe un phénomène identique à un niveau moindre. Néanmoins, quelle que soit l'hormone de croissance utilisée, on note une importante variabilité des valeurs enregistrées, les coefficients de variation pour les temps I et 2 heures après les traitements PGH et BGH sont importants, souvent de l'ordre de 30 à 45 p. Ioo. Cette observation sur la variabilité des phénomènes observés nous conduit à la plus grande prudence en ce qui concerne l'interprétation des résultats obtenus sur les taux plasmatiques en glucides réducteurs.

On constate également que la glycémie est maintenue à des valeurs subnormales chez les animaux privés d'hypophyse quand ils sont nourris ad libitum : les témoins hypophysectomisés ont eu dans notre expérience une glycémie moyenne de $125 \mathrm{mg}$ pour roo $\mathrm{ml}$ de plasma, pour les temps 0 , tandis que 5 animaux normaux de même âge avaient une moyenne de $\mathrm{I} 39 \mathrm{mg}$ pour Ioo $\mathrm{ml}$ de plasma. Nous avons par ailleurs 
montré dans un travail non publié, que des lapins hypophysectomisés soumis à un jeûne de 24 heures présentaient des glycémies très affaiblies de l'ordre de $80 \mathrm{mg}$ pour Ioo $\mathrm{ml}$ de plasma tandis que chez des lapins normaux, ce même jeûne était sans effet sur le taux en glucose du plasma. Cette constatation pose le problème du maintien de la glycémie chez le Lapin hypophysectomisé.

Un autre point mérite d'être discuté ici; il concerne les effets somatotropes des traitements chroniques à la GH. Le tableau 3 où sont comparés les gains de poids quotidiens moyens entre les animaux traités à la $\mathrm{PGH}$, à la $\mathrm{BGH}$ et leurs témoins hypophysectomisés sans traitement montrent que dans aucun cas on n'observe de gain de poids notable. Les animaux traités à la PGH perdent même en moyenne I3 $\mathrm{g}$ par jour. Cela pourrait être expliqué par le fait que leurs réserves adipeuses se trouvent réduites comme nous l'avons déjà montré. Le très faible gain enregistré pour les animaux traités à la $\mathrm{BGH}$ ne semble pas avoir de signification particulière.

Il apparaît donc nettement que, ni la PGH, ni la BGH, ne sont capables d'induire une accélération du gain de poids chez le Lapin hypophysectomisé à l'âge de roo jours et dont l'arrêt de croissance avait été constaté, ce qui corrobore les résultats déjà obtenus dans un précédent travail (VÉzINHET, I968). Cette absence d'effet sur la croissance peut s'expliquer de diverses façons : soit par le rythme des injections adopté ici qui pourrait être insuffisant, soit par la très haute spécificité des hormones soma-

TABLEAU 3

Comparaison des gains de poids entre animaux hypophysectomisés traités à la $P G H$ et à la $B G H$ et leurs témoins non traités

\begin{tabular}{|c|c|c|c|}
\hline \multirow[b]{2}{*}{ Poids des animaux } & \multicolumn{3}{|c|}{ Traitement } \\
\hline & $\begin{array}{l}\text { Hypophysectomisés } \\
\text { traités PGH }\end{array}$ & $\begin{array}{c}\text { Hypophysectomisés } \\
\text { traités BGH }\end{array}$ & $\begin{array}{c}\text { Témoins } \\
\text { hypophysectomisés } \\
\text { ClNa } 9 \text { p. } 1000\end{array}$ \\
\hline $\begin{array}{l}\text { Poids au début } \\
\text { du traitement }\end{array}$ & $\begin{array}{c}2661 \pm 284 \\
n=5\end{array}$ & $\begin{aligned} 2602 & \pm 197 \\
n & =4\end{aligned}$ & $\begin{array}{c}2845 \pm 334 \\
n=6\end{array}$ \\
\hline $\begin{array}{l}\text { Poids à la fin } \\
\text { du traitement }\end{array}$ & $\begin{aligned} 2506 & \pm 368 \\
n & =5\end{aligned}$ & $\begin{array}{c}2647 \pm 389 \\
n=4\end{array}$ & $\begin{aligned} 2796 & \pm 305 \\
n & =6\end{aligned}$ \\
\hline $\begin{array}{l}\text { Gain de poids } \\
\text { quotidien moyen }\end{array}$ & -13 & +4 & -4 \\
\hline
\end{tabular}

totropes; mais alors les résultats que nous avons obtenus tant sur le métabolisme lipidique que sur celui des glucides sembleraient éliminer l'hypothèse d'une inactivité liée à la spécificité, si l'on admet comme RABEN (r965) que tous les effets de la GH sont imputables à une seule molécule. Une deuxième hypothèse, celle de LEVINE et LUFT (I964) postulant l'existence de deux hormones physiologiquement distinctes, la somatotrope proprement dite $(\mathrm{STH})$ et le facteur adipocinétique $(\mathrm{AK})$ permettrait d'admettre que 1'une soit très spécifique (STH) et l'autre beaucoup moins, ou pas du tout (AK). TRygstad et Foss (I968) pensent qu'une seule molécule de GH 
pourrait renfermer une fraction à pouvoir somatotrope (STH) et une autre fraction à pouvoir lipolytique (LMF). Cette dernière hypothèse pourrait concilier les deux précédentes et dans ce cas nous pourrions concevoir qu'à l'intérieur d'une même molécule deux séquences successives pourraient avoir des comportements différents quant à leur spécificité.

Reçu pour publication en février 1972.

\section{REMERCIEMENTS}

Les auteurs doivent remercier le docteur A. E. Wilhelmi (Woodruff Medical Center of Emory University, Atlanta Georgia) et le National Institute of Health pour le don de GH porcine. Leurs remerciements s'adressent également au Medical Research Council, W. H. O. (Mill Hill, London, N. W. 7) qui nous a permis d'étalonner en UI la préparation commerciale de BGH dont nous disposions.

\section{SUMMARY}

\section{EVOLUTION OF FREE FATTY ACID AND REDUCTION SUGAR CONTENT \\ IN THE PLASMA OF THE HYPOPHYSECTOMIZED RABBIT DURING TREATMENT WITH PIG AND CATTLE SOMATOTROPIC HORMONES}

The effect of repeated BGH and PGH injections on the evolution of free fatty acids and carbohydrate content in the plasma of hypophysectomized rabbit is studied. Metabolite dosages were done on samples of venous blood between o hours before the treatment, and then I, 2, 5 , and 9 hours. after injection of the hormone under consideration.

Glycemia : PGH administration has a hypoglycemiant effect on the hypophysectomized rabbit, fed ad libitum, which is observed on to two hours after injection. This effect is not followed by hyperglycemia. Results obtained with BGH are similar but less intense.

Free fatty acid content: a high elevation is observed with $\mathrm{PGH}$ at one and two hours after injection, and the content drops to normal at the end of 9 hours. BGH has no particular effect. When some animals are sacrificed, adipose storage deposits (estimated from perirenal fat) seem clearly reduced in hypophysectomized rabbits treated with PGH as related to hypophysectomized controls treated with CINa 9 p. I ooo.

There was no variation in general ponderal growth.

\section{RÉFÉRENCES BIBLIOGRAPHIQUES}

Altszuller N., Rathgeb I., Winkler B. N., de Bodo R. C., I968. The effects of growth hormone on carbohydrate and lipid metabolism in the dog. Ann. N. Y. Acad. Sci., 148, 441.

Dole V. P., Meinertz H., I960. Microdetermination of long chain fatty acids in plasma and tissues. J. biol. chem., 235, 2595-2599.

Ellis S., Grindeland R. E., Nueke J. M., Callahan P. X., I968. Isolation and properties of rat and rabbit growth hormones. Ann. N. Y. Acad. Sci., 148, 328.

Engel H. R., Bergenstal D. M., Nixon W. E., Patton J. A., 1959. Effect of human growth hormone on unesterified fatty acid and plasma amino acid nitrogen in man. Proc. Soc. exp. Biol. Med., 100, 699.

Engel H. R., Hallman L., Siegel S., Bergenstal D., I958. Effect of growth hormone on plasma unesterified fatty acid and level of hypophysectomized rats. Proc. Soc. exp. Biol. Med., 98, 753 .

Goodman H. M., KNoBiL E., 1959. Effect of $\mathrm{pH}$ and route of administration on the fatty acid mobilizing activity of growth hormone solutions. Endocrinology, 65, 977. 
Greenbaum A. L., Glascock R. F., I957. The synthesis of lipids in the livers of rats treated with pituitary growth hormone. Biochem. J., 67, 360 .

KNobil E., GReEP R. O., I959. The physiology of growth hormone with particular references to its action in the rhesus Monkey and the "species specificity" problem. Recent. Prog. Horm. Res., 15, I-69.

LEvine R., LUFT R., I964. The relation between the growth and diabetogenic effects of the so called growth hormone of the anterior pituitary. Diabetes, 13, 65 I-655.

Nelson N., I944. A photometric adaptation of the Somogyi method for the determination of glucose. J. biol, chem., 153, 375 .

Raben M. S., I959. Human growth hormone. Recent. Prog. Horm. Res., 15, 7I.

RABEN M. S., I965. Growth hormone : anabolic and anticatabolic agent. Diabetes, 14, 374.

Raben M. S., Hollenberg C. H., I958. Effect of growth hormone on fatty acids. J. clin. Invest., 37, 922.

VÉzinhet A., 1968. Effet de l'hypophysectomie sur la croissance pondérale du Lapin. C. R. Acad. Sci., 266, 2348-235I.

TRYGSTAD O., Foss I., I968. The lipid mobilizing effect of some pituitary gland preparations. IV. Subdivision of a human growth hormone preparation into a somatotrophic and an adipokinetic hyper. glycemic agent. Acta Endocr. Copenh., 58, 295-317.

Wilhelmi A. E., in : Smith R. W., Gaebler O. M., I955 and Long C. N. H., 59-104. Hypophyseal growth hormone. Nature and Action. Mc Graw-Hill, New York.

Winkler B. N., Steele R., Altszuller N., Dunn A., de Bodo R. C., rg62. Effects of growth hormone on FFA metabolism. Fed. Proc., 21, r98. 